Théologiques

Théologiques

\title{
Le mysticisme silencieux des Sekani de la Colombie-Britannique (Canada). L'individualisation du mythique et la territorialisation de l'individualité
}

\section{Guy Lanoue}

Volume 16, numéro 2, 2008

L'individualisation de la relation religieuse

URI : https://id.erudit.org/iderudit/001718ar

DOI : https://doi.org/10.7202/001718ar

Aller au sommaire du numéro

\section{Éditeur(s)}

Faculté de théologie et de sciences des religions, Université de Montréal

\section{ISSN}

1188-7109 (imprimé)

1492-1413 (numérique)

Découvrir la revue

Citer cet article

Lanoue, G. (2008). Le mysticisme silencieux des Sekani de la Colombie-Britannique (Canada). L'individualisation du mythique et la territorialisation de l'individualité. Théologiques, 16(2), 123-139.

https://doi.org/10.7202/001718ar

\section{Résumé de l'article}

Les Sekani de la Colombie septentrionale (Canada) étaient des chasseurs nomades qui vivaient, jusqu'aux années 1970, en petits groupes dispersés et autonomes sur le plan économique. Dans cet article, j'examine comment l'hyperindividualité qui accompagne ce style de vie est utilisée pour définir un champ de silence qui devient la base de la communauté politique. Pour ce faire, les Sekani déplacent l'agir individuel vers un domaine apparemment " surnaturel ", encourageant les chasseurs à prendre contact avec les esprits tutélaires. Le silence qui entoure de tels contacts déclenche des commérages " secrets " qui aboutissent à l'identification d'un homme fort capable ainsi d'assumer le statut de chef gestionnaire du territoire national. 


\title{
Le mysticisme silencieux des Sekani de la Colombie-Britannique (Canada). L'individualisation du mythique et la territorialisation de l'individualité
}

\author{
Guy LANOUE* \\ Département d'anthropologie \\ Université de Montréal
}

\section{Introduction}

Dans cet article, je veux présenter une étude de cas où le contact individuel avec le mystique est conçu à la fois de façon négative et positive, où la tension suivant le contact avec le domaine invisible (conçu comme parsemé de forces primordiales) mène à la marginalisation de l'individu qui, paradoxalement, devient l'agent de la création de la communauté gestionnaire du territoire «national ». En particulier, le discours qui lie l'individu à la communauté émerge de l'acquisition du pouvoir individuel à travers le lien au mythique.

Poussés, par leur système d'exploitation de l'environnement naturel, à avoir peu de contact entre les petits groupes de chasse, les Sekani ont un sens relativement faible de la communauté politique. Cependant, ils reconnaissent qu'ils doivent trouver un moyen pour y arriver, car ils revendiquent approximativement dix fois plus de territoire par rapport à la superficie qu'ils utilisent habituellement dans une saison de chasse. Cette

\footnotetext{
Guy Lanoue est professeur titulaire en anthropologie à l'Université de Montréal. Ses recherches ont longtemps porté sur les Sekani semi-nomades de la ColombieBritannique septentrionale, ainsi que sur les paysans, les apprentis et la bourgeoisie romaine. Récemment, il a lancé un projet de recherche sur le nouveau cosmopolitisme et la disparition de la classe moyenne européenne. Parmi ses publications, on trouve (avec Jean-Marc Desgent) Errances. Comment se pensent le Nous et le Moi dans l'espace mythique des nomades septentrionaux sekani, Ottawa, Musée canadien des civilisations (Mercury Series, Cultural Studies Paper 78), 2005.
} 
situation crée des conditions politiques difficiles, car leurs voisins peuvent être tentés d'envahir et d'occuper les espaces vides. C'est donc uniquement en démontrant à leurs voisins qu'ils sont relativement unis sur la question du territoire national qu'ils assurent la survie des petits groupes de chasse et, donc, des individus qui les composent. Pour ce faire, ils utilisent la dimension mythique, car ils développent ainsi une notion de la communauté qui ne contredit pas l'autonomie individuelle, puisqu'elle est projetée sur la dimension mythique et non basée sur les accomplissements individuels.

Pour les Sekani, un peuple athapascan du Nord canadien, naguère des chasseurs semi-nomades ${ }^{1}$, la dimension mystique "contamine» certains hommes pour les transformer en individus surpuissants ${ }^{2}$, leur permettant de servir de pivot autour duquel se concrétise la communauté qui, autrement, n'a qu'une existence fragile et éphémère, car les technologies de chasse poussent les personnes à être isolées les unes des autres pour une grande partie de l'année, ce qui réduit l'intimité sociale et encourage l'individualisme. Ironiquement, la surpuissance de tels individus marqués par les forces de la dimension invisible n'est un agent politique efficace que si elle se manifeste indirectement.

Les Sekani possèdent une pensée très nuancée à propos de la dimension mythique et de ses qualités, mais il est clair que leurs notions du transcendantal sont une expression de leur désir de créer et de définir une communauté utilisant des moyens qui ne menacent aucunement l'autonomie

1. Ils étaient semi-nomades et largement dépendants des produits de la chasse jusqu'aux années 1960. Leur territoire se situe dans le bassin hydraulique de l'océan Arctique, dans la partie septentrionale des montagnes Rocheuses de la Colombie-Britannique, où ils vivent en trois petits villages isolés. J'ai vécu presque deux ans avec eux à la fin des années 1970. Désormais, ils sont plus sédentaires que nomades, après que le gouvernement provincial a construit un barrage hydroélectrique sur le fleuve Peace en 1968, inondant une grande partie de leur territoire et ouvrant la zone à la colonisation euro-canadienne. En conséquence, plusieurs aspects des connaissances ici décrites ont disparu de la mémoire collective. J'adopte ici le "présent ethnographique ", car ces connaissances étaient toujours en vigueur parmi les aînés quand je les ai connus. À ma connaissance, je suis un des derniers dépositaires de telles connaissances.

2. Ici, je ne puis approfondir la discussion du phénomène de la possession. J'utilise le mot «contamination» pour me rapprocher des croyances sekani et pour éviter la confusion avec des études de cas plus connues où l'individu est en contact avec des esprits qui assument une forme bien définie. Pour les Sekani, le contact s'effectue non avec des entités, mais avec des forces primordiales dont l'unique manifestation est dans «l'esprit » de l'animal. Plus important, ce contact n'éclipse pas la personnalité de l'individu, sauf pour quelques circonstances rares. 
individuelle dont ils ont besoin pour assurer leur survie. Bref, ils déplacent et projettent sur le mythique une pensée politico-territoriale marquée par une contradiction fondamentale entre, d'une part, la solidarité de la communauté face à leurs voisins toujours désireux d'infiltrer les parties non utilisées du territoire sekani, et, d'autre part, l'autonomie individuelle qui leur permet de réagir rapidement et de façon efficace aux circonstances environnementales toujours en évolution.

Il n'est donc pas surprenant que les forces primordiales de la dimension mystique soient considérées comme «naturelles », car elles sont une partie intégrale de la structure du cosmos et liées à la définition de la communauté. En revanche, l'humain est « infranaturel », car, doué uniquement d'une culture dont l'aspect politique est forcément faible et éphémère, il n'a pas normalement accès aux forces primordiales qui parsèment cette dimension. C'est uniquement par la contamination de l'individu à la recherche du contact avec ces forces que les Sekani réussissent à repérer des différences individuelles importantes, lesquelles définissent les acteurs clés capables de concrétiser une hiérarchie "spirituelle » qui gère et exprime leurs revendications territoriales et, donc, leur identité en tant que communauté. Ironiquement, l'individu surpuissant est vu comme partiellement déséquilibré, car il est doué d'une force transcendantale apte à contrôler ses actions s'il ne prend aucune précaution. Il est en conséquence partiellement aliéné de la communauté. Nous connaissons les cas extrêmes de "contamination" sous la rubrique de "sorcier ", mais il est significatif que de tels cas puissent aussi mener à une forme de cannibalisme symbolique (wechuge, qui transforme une personne en monstre cannibale; l'exemple mieux connu est windigo, un phénomène semblable parmi les peuples algonquins du Nord canadien), où l'anthropophagie est un symbole puissant d'une communauté (imaginée) tellement solidaire, qu'elle en devienne autophage et anéantisse toute forme d'individualité (voir Lanoue et Ferrera 2004)3.

Pour mieux comprendre cette pensée «religieuse » des Sekani, ne possédant ni dieux ni rituels unificateurs, il est nécessaire de décortiquer leur façon de définir l'individualité. La construction de l'individualité crée la base symbolique de la communauté «faible ", qui devient la force motrice pour les tentatives de contacter les forces primordiales de la dimension mystique.

3. Je ne peux approfondir ici l'importance du cannibalisme symbolique (il n'y a aucune preuve qu'il ait été pratiqué) sauf pour souligner que l'incorporation de la chair de l'autre est une métaphore puissante pour la production de la solidarité sociale. Voir Turner (1978). 
À leur tour, ces contacts deviennent l'engin qui anime la communauté politique dont le but est de gérer le territoire national.

\section{L'individualité et les « autres»}

À la base des interactions sociales sekani est le principe de respect de l'autonomie d'autrui, et ce, dès la plus tendre enfance ${ }^{4}$. Pour les nomades septentrionaux, il n'existe aucune façon acceptable d'imposer ou même de transmettre ses connaissances à un autre. Le savoir d'un individu est considéré comme inutile en dehors du contexte dans lequel il a été créé, c'està-dire que les connaissances de l'environnement naturel et du milieu social ont un sens uniquement en fonction des circonstances qui entourent l'agir. Nous pourrions dire que les connaissances sont encodées pour l'individu qui les a créées et qu'il y a ainsi reconnaissance des limitations de l'agir individuel. Ce qui devient vrai pour un individu n'est pas nécessairement valide pour un autre, puisque les connaissances sont vues comme enracinées dans les expériences particulières de chaque individu. En ce qui concerne les techniques (p.ex.: fendre le bois), l'agir adéquat est vu comme la conséquence d'une réflexion sur le monde passant par le transcendantal. Il n'est pas imposé par des contraintes d'ordre purement matériel. Autrement dit, si on ne sait pas bien fendre le bois ou tirer au fusil, ce n'est pas dû au fait que la hache ne soit pas aiguisée ou que le fusil soit vieux, mais c'est parce que l'individu ne sait pas s'adapter aux circonstances. En général, cette attitude envers les connaissances repose sur la croyance que la vérité, au sens platonicien du terme, n'existe que dans la dimension mythique.

De plus, l'enseignement formel est considéré comme une atteinte aux bonnes manières, puisqu'une telle action placerait l'enfant dans l'obligation d'écouter ou, dans le cas d'un adulte, d'explicitement rejeter ses propres connaissances comme appartenant à un autre cadre expérimental. Les parents créent des situations favorisant l'apprentissage et la formation des enfants en les exposant à un ensemble de situations dans lesquelles ils observeraient un comportement judicieux et efficace, et arriveraient à des conclusions souhaitables. Autrement dit, il n'y a aucun système formel de transmission de connaissances, ce qui renforce l'autonomie individuelle tant nécessaire pour affronter l'environnement impardonnable.

4. Voir l'excellente discussion de ce point en Goulet (1994); ici, je me contenterai de fournir quelques exemples. 
Le respect de l'autonomie de l'autre est si profondément enraciné dans les valeurs, qu'un individu interroge rarement un ami ou un parent sur ses déplacements ou ses intentions; cela le mettrait dans l'obligation de répondre, voire de mentir, s'il ne veut communiquer certains détails. D'un autre côté, quand deux chasseurs se rencontrent, ils échangent volontairement des informations concernant leurs déplacements récents afin d'enrichir les connaissances géographiques indispensables au succès de la chasse. Selon la même logique, on n'interrogera pas directement un chasseur sur sa récolte, même si l'on s'attend à une distribution généreuse de gibier (toujours en respectant les liens de parenté et d'alliance). Si une personne n'est pas généreuse, on ne peut l'obliger à le devenir qu'en la critiquant indirectement. Le respect de l'autre se manifeste par une politesse assez formelle et par un style de rhétorique indirecte, particulièrement lors de rencontres politiques formelles au cours desquelles pourraient se manifester des différences de pouvoir et de statut.

La politesse envers l'autre typique de la vie sociale est le signe d'un respect extrême de son individualité. Dans le même ordre d'idées, les Sekani n'ont pas d'heures fixes pour les repas et mangent quand ils ont faim (il y a toujours quelque chose de prêt s'il y a de la nourriture dans la maison ou dans le camp de chasse). La majorité des parents n'empêchent pas leurs adolescents de consommer des aliments nocifs, y compris de l'alcool. Si un parent qui consomme de l'alcool désire empêcher ses enfants de développer cette habitude, il cachera l'alcool plutôt que de leur en défendre la consommation. Un parent cesserait de fréquenter les personnes qui en consomment, et pourra même déménager ou changer ses habitudes afin de ne plus risquer de mettre ses enfants en présence d'une si mauvaise influence. À maintes reprises, on peut entendre des dénonciations concernant la consommation d'alcool sans que celles-ci se transforment en action.

Le respect de l'individualité de l'autre n'est pas limité aux jeunes, mais concerne aussi les personnes âgées. Chacun est considéré comme responsable de sa propre vie. L'hiver, les personnes âgées restent parfois seules dans leur maison sans avoir facilement accès à du bois de chauffage ou à de la nourriture. De temps à autre, un jeune peut passer afin de s'assurer qu'ils ont de quoi survivre; cependant, ces visites ne semblent ni fréquentes ni systématiques. S'il est vrai que les personnes âgées sont respectées, il est aussi vrai qu'elles sont souvent ignorées; ce qui est tout à fait conséquent avec leur conception du respect de l'autonomie de l'autre. L'idée que l'on respecte les anciens pour leurs connaissances ressemble davantage à une 
projection exotique blanche qu'à une réalité autochtone, bien que plusieurs Amérindiens, surtout les jeunes les plus politisés, semblent l'avoir adoptée récemment.

Cette «surindividualité » est à la base de la collaboration entre partenaires de chasse et entre hommes et femmes, bien que la tension ne soit pas absente de la vie quotidienne. Les contradictions à la base de la tension sociale ont toujours été nécessaires au mode de vie des chasseurs, puisque celles-ci appuient l'individualité dont dépend le chasseur dans sa recherche du gibier et, donc, affaiblissent l'image de la communauté politique. La jalousie est en effet chose courante, surtout si le succès à la chasse ne s'accompagne pas d'actes publics de générosité (la pire insulte que l'on puisse faire à une personne est de la traiter d'avare). En général, la générosité étant une des valeurs publiquement appréciées, elle cristallise des opinions négatives et positives et devient donc une force motrice pour la création de la communauté (comme l'est l'inverse de tels commérages, c.-à-d. le silence qui entoure les pouvoirs du chasseur).

Des factions existent au sein de la communauté; elles sont liées à la politique locale, mais elles sont souvent tournées vers la dimension mythique des Animaux-protecteurs 5 . Les maladies ou les échecs sont invariablement interprétés comme le résultat d'actes de sorcellerie, mais ils représentent en réalité des confrontations dans la dimension mythique entre les hommes et leurs Animaux-protecteurs. Par conséquent, les plus chanceux ou les plus habiles devront faire preuve de beaucoup de générosité puisque leur bonne chance n'est aucunement fruit de leur talent.

Il existe aussi une tension entre les hommes et les femmes, qui n'est pas entièrement attribuable aux problèmes liés à la fragilisation récente de la culture sekani par les pratiques euro-canadiennes. Les deux sexes occupent des espaces sociaux relativement distincts, sans que l'un soit soumis à l'autre; la catégorie "femme» est marquée sémiotiquement de façon très différente de celle d' «homme». Par exemple, il est vrai que les femmes sont en général responsables des tâches dites domestiques comme la cuis-

5. Dans la littérature ethnographique, les Animaux-protecteurs sont parfois appelés «esprits tutélaires», mais les spécialistes des peuples athapascan évitent cette expression. Le mot «esprit» nous renvoie à un concept occidental et cartésien du rapport corps-esprit, quand en fait il s'agit de la force primordiale présente dès la création du monde et toujours présente dans les animaux. $L^{\prime}$ "Animal-protecteur» est en fait la dimension invisible, mais surpuissante de l'animal. Pour distinguer celle-ci de la dimension biologique des animaux, nous utiliserons «Animaux» ou «Animauxprotecteurs», avec la majuscule. 
son de la nourriture. Les femmes fendent le bois et participent à plusieurs activités difficiles - récolte des baies, chasse de petits animaux, préparation des peaux - sans oublier la prise en charge des enfants (dans une maisonnée ou dans un camp, cette responsabilité est partagée par toutes les femmes et les enfants sont habitués dès un très jeune âge à se débrouiller; il n'y a pas de "mère de famille ", puisqu'il n'existe pas de famille en tant que telle ${ }^{6}$ ). Cependant, les activités qui marquent le féminin et le masculin ne sont pas hiérarchisées. S'il est vrai que la galanterie européenne est absente $^{7}$, il est aussi vrai que les hommes ont en général tendance à considérer les femmes comme leurs partenaires et non comme des êtres inférieurs ou dépendants. Dans le même ordre d'idées, chaque femme est responsable de sa vie sexuelle. Semble absente l'idée que les hommes peuvent ou ont à contrôler la sexualité des femmes: tous sont responsables de leur survie, de leur qualité de vie, incluant leur sexualité.

Bref, les règles du savoir-vivre sekani sont complexes, comme dans toutes les sociétés, mais n'aboutissent pas à la production de régularités sociales ressemblant à des institutions ou à des valeurs explicites pour deux raisons : 1) essentiellement, il n'y a que deux valeurs partagées, la générosité et le silence qui transforment chaque discours en commérage; ces deux s'annulent, dans un sens, car la générosité renforce la solidarité, mais le silence appuie l'individualité ; 2) les pratiques de vie sekani encourageaient davantage l'individualité et donc l'individuation, qui agirait contre la formation de la communauté déjà fragilisée par la dispersion des groupes de chasse.

Les deux conclusions qui émergent de ce bref survol de la vie sociale sekani - les espaces institutionnels politisés n'existent pas et les valeurs et croyances publiquement affirmées ne servent pas forcément à renforcer la solidarité sociale - nous suggèrent que la communauté sekani ne trouve pas son signifié dans les pratiques concrètes de la vie sociale. En résumé, connaître le monde signifie le contrôler; cependant, le savoir est le fruit de l'interaction entre l'individu et les deux dimensions de l'univers, le vécu et

6. Des regroupements de famille existent dans le sens où les Sekani, s’ils sont interrogés par un Euro-canadien, sont capables de les produire, car ils savent que cette catégorie est importante pour les Blancs, surtout pour ceux et celles qui travaillent dans les services sociaux (et donc, qui sont potentiellement dangereux, car ces derniers peuvent suggérer à un tribunal que les soins fournis aux jeunes sont inadéquats).

7. Dont le sous-texte sert selon toute vraisemblance à marquer les femmes comme faibles et dépendantes. 
le mythique. Cette deuxième dimension n'est connue que par l'individu qui, ironiquement, devient l'agent de la construction du monde social.

\section{Le mystique et la naissance de la communauté}

Avant qu'ils ne deviennent semi-sédentaires dans la période $1940-1950^{8}$, les Sekani vivaient en petits groupes de chasse séparés les uns des autres par de grandes distances. Ces composants des trois bandes ${ }^{9}$ sekani (dont chacune était composée de 8-10 groupes de chasse) se regroupaient uniquement pendant les deux ou trois mois de l'été ${ }^{10}$, près des fleuves et des lacs situés au cœur de leur territoire pour en exploiter les ressources halieutiques, les seules capables de soutenir une population de 150-200 individus (les animaux chassés par les Sekani, surtout l'orignal et le caribou, sont en général aussi individualistes et dispersés qu'eux $\left.{ }^{11}\right)$. En automne, après un échange de personnes et un partage d'informations, ils se séparaient de nouveau, chaque petit groupe allant à une zone d'exploitation parfois éloignée de plus de 100 ou $150 \mathrm{~km}$ par rapport au point de regroupement estival. Le territoire exploité par chaque groupe de chasse était de 200 à $300 \mathrm{~km}^{2}$. Étant donné qu'existaient une dizaine de tels groupes par bande, ceci signifie que seulement 2000 à $3000 \mathrm{~km}$ carrés étaient exploités, un pourcentage modeste ${ }^{12}$ de la superficie revendiquée de $10000 \mathrm{~km}^{2}$. Ils étaient obligés de revendiquer plus de territoire qu'ils n'en utilisaient dans une saison pour anticiper les changements environnementaux et écologiques imprévisibles, avec comme résultat que leur densité démographique plutôt faible créait ainsi une situation où il devenait difficile, voire impossible, d'invoquer la solidarité mécanique pour former la communauté.

8. La sédentarisation ne s'est pas imposée selon un seul programme pour les Sekani. Le groupe septentrional a poursuivi les rythmes traditionnels de déplacement jusqu'à la fin des années 1960 .

9. Dans la littérature populaire, tels regroupements sont parfois nommés «tribus», mais il s'agit en fait de bandes, car ces dernières catégories ne sont pas douées de système clanique, un trait dont les qualités font office de matrice pour une forme d'organisation sociale et politique entièrement différente.

10. À des lieux connus, dès le début du $\mathrm{xx}^{\mathrm{e}}$ siècle, sous les noms de Fort Ware (nord), Fort Grahame (centre), et McLeod Lake (sud).

11. Ce chiffre, ainsi que les détails présentés ici en synthèse, sont tirés de l'analyse proposée par Lanoue (1992).

12. Pour les chasseurs du Canada septentrional, une telle superficie est assez petite. Traditionnellement, les peuples du Nord revendiquaient des territoires de 20000 à $30000 \mathrm{~km}^{2}$. Le territoire sekani était limité aux vallées des montagnes Rocheuses. 
Autrement dit, plus grand était le territoire revendiqué, plus il y avait de pression pour que les groupes de chasse se déplacent sur toutes les parties du territoire pour démontrer qu'ils en étaient les propriétaires. Le déplacement était censé envoyer le message selon lequel même une zone temporairement vide devait être respectée par les groupes avoisinants. Les petits groupes de chasse étaient censés changer de zone tous les deux ou trois ans pour se déplacer vers des zones relativement inconnues et faisant face à la possibilité que ces zones délaissées fussent possiblement utilisées par leurs voisins.

Dans ce contexte, il devient impératif de convaincre ces petits groupes de participer au système de déplacement et d'affronter les incertitudes des zones non occupées, toujours en respectant l'autonomie des individus. Comment définir un leader capable d'organiser ce déplacement qui se heurtait directement au pouvoir individuel, et surtout comment définir l'obligation morale d'obéir au chef? Les Sekani ont choisi de lier ce projet politique à la vision d'une dimension mystique, qui, cependant, semble loin d'être surnaturelle; en fait, elle est vue comme naturelle, toujours parsemée de forces primordiales présentes dès la création de la terre. La primauté de telles forces signifie que la société et l'humain sont des catégories «infranaturelles ", c'est-à-dire qu'ils sont relativement impuissants devant la dimension mystique ${ }^{13}$.

Le mystique constitue l'agent et le lieu où les individus cherchent le contact avec ce pouvoir transcendantal afin d'augmenter leur capacité de chasse, c'est-à-dire qu'ils chercheraient à se mettre sur un pied d'égalité avec les animaux toujours doués d'une force primordiale. À la différence des humains, les animaux survivent dans le même environnement sans langue, sans apprentissage, et surtout sans outils. Ceci constitue, aux yeux des Sekani, la preuve définitive que cette force primordiale est toujours présente dans les animaux, et que les humains, ayant besoin de ces «technologies »

13. Il y a des parallèles ailleurs, évidemment. Par exemple, le mana est le concept polynésien du pouvoir spirituel qui caractérise tous les objets et les personnes. Le mot dérive d'une langue mélanésienne qui décrit un pouvoir anthropomorphique (voir Codrington 1972). L'orenda est un concept iroquoien (incluant les Hurons des Grands Lacs) très semblable à l'idée algonquienne du manitou ( «Grand»), de même qu'au wakan des Sioux (wakanda = "Grand»), au maxpe des Crow de l'Ouest américain, et à l'elima des Nkundu et les Bambuti du Bassin du Congo en Afrique. Le mot orenda semble lié au mot mohawk signifiant " chanson», qui suggère que la performance des rituels fut d'une importance capitale pour conserver et contrôler cette force (voir Bierhorst 1985 et Hultkrantz 1967). 
culturelles, sont privés de tels pouvoirs ${ }^{14}$. En rentrant en contact avec la dimension transcendantale des animaux, les hommes peuvent acquérir une partie de la force primordiale toujours présente dans l'animal.

Cette force, comme je l'ai noté, est toujours active. Les Sekani croient que c'est uniquement la forme biologique actuelle de l'animal qui l'empêche de se manifester. Les aspects biologiques des animaux, tels qu'on les connaît aujourd'hui, sont le résultat de l'intervention d'une entité un peu " arnaqueuse ", modérément stupide et entièrement grossière, nommée le Transformateur ${ }^{15}$. Avant l'arrivée de ce dernier, les animaux naturellement puissants vivaient leur supériorité : ils étaient surdoués sur le plan biologique et culturel. Pour leur part, les humains faibles étaient incapables de chasser de façon efficace. Ils étaient souvent la proie de ces Animaux surpuissants. Le Transformateur, constamment à la recherche de nourriture et de relations sexuelles, était souvent incapable de réaliser ses plans, dû à sa stupidité et à son manque de prévoyance. À la suite de l'échec de ses plans, il transforme par rancune ou par inadvertance l'aspect biologique des Animaux, les rendant matériellement incapables de manifester leur pouvoir surhumain (surtout signalé par la perte de leur langue).

À noter que le Transformateur ne transforme ni n'aide les humains. Le héros lévi-straussien Asdiwal (Lévi-Strauss 1973) aidé par son père surnaturel, le Soleil, à prendre sa place de droit dans la hiérarchie tsimshian est symboliquement très loin des idées sekani du soi-disant surnaturel, même si en réalité Asdiwal vivait à peine cent kilomètres à l'ouest du territoire sekani. Dans le rapport d'inégalité entre humains et Animaux surpuissants, le Transformateur réduit la capacité d'agir des Animaux, ce qui permet aux humains d'exprimer leurs propres talents. À noter que cette idée d'affaiblir une catégorie sans l'éliminer est typique de la pensée sekani visant à contourner l'individualité surpuissante sans l'anéantir.

Afin de prendre contact avec cette dimension, les humains d'aujourd'hui doivent se transformer en gibier symbolique (la soi-disant quête de

14. Dans nos conversations, les Sekani ont rarement utilisé ce mot dans leur langue ou en Anglais. Parmi les Athapascans, le "pouvoir» est appelé zhaak ("grâce ", Legros 1999) par les Tutchones septentrionaux, in’kon (Helm 1994) par les Dogrib, nitsit (McDonnell, communication personnelle) par les Kaska. Voir Sharp (1987 et 1988) pour une discussion du même concept parmi les Chipewyan et Ridington (1988a) pour les Beaver.

15. Étant donné ses traits plutôt négatifs, j’hésite à utiliser l'expression mieux connue de « héros culturel». 
vision): se déplaçant dans la forêt et demeurant immobiles, sans armes, sans nourriture, les hommes tentent d'inciter l'animal à manifester la dimension de sa nature désormais "silencieuse", mais toujours surpuissante. Autrement dit, si l'animal s'approche de l'humain immobile, à jeun et sans armes, c'est que la dimension primordiale a saisi le contrôle de l'animal qui, par sa nature biologique désormais limitée, serait plutôt porté à s'enfuir. Selon les Sekani, le contact prend la forme symbolique d'une conversation, c'est-à-dire d'un échange : l'humain sous l'apparence de gibier se "donne » à l'animal et l'animal partage quant à lui son pouvoir primordial. C'est l'inverse du rapport quotidien, où l'humain offre le respect en contre-don pour la vie de l'animal, dont la supériorité innée signifie qu'il ne peut pas être tué sans se sacrifier volontairement. Dans le cas du contact avec la dimension mystique, l'humain, gibier métaphorique et donc «ingéré » par l'animal, devient une partie symbolique de l'animal, qui le met naturellement en contact avec la force primordiale de l'animal.

Doué d'une force supérieure, à la suite de la rencontre, l'individu devient non seulement un meilleur chasseur, mais il acquiert également un statut supérieur grâce à ses nouveaux pouvoirs qui sont attestés par sa générosité, faisant des dons importants de gibier aux personnes moins fortunées dans leurs tentatives de contacter la force primordiale. Pour les Sekani, seul le don de gibier est significatif, car, étant partiellement transformé en animal, le chasseur donne une partie de lui-même ${ }^{16}$. Il est impossible de donner des objets quotidiens, car les personnes apparentées ont le droit de prendre quoi que ce soit, sans la permission du propriétaire. On peut donner uniquement ce qui nous appartient, c'est-à-dire une partie de nous-mêmes.

Le statut accordé au chasseur chanceux découle autant de la crainte et de l'envie des autres. Les individus surdoués grâce au contact avec la dimension primordiale sont toujours potentiellement dangereux : comme le sont les femmes qui sont naturellement douées de pouvoir, ce qu'atteste leur sang menstruel — signe de leur force reproductrice —; comme le sang versé par l'animal est signe de la survie biologique de l'homme. Le statut rehaussé du chasseur contaminé par le transcendantal, dans une société largement

16. Comme plusieurs aspects des valeurs sekani qui affirment et nient l'individualité, le don du gibier doit normalement se faire avant de rentrer dans la communauté ou au camp de chasse car, en rentrant dans la sphère domestique, le chasseur fait don de sa prise à son épouse, qui la distribue aux parents et aux amis. 
égalitaire, est en partie accordé par crainte de ses pouvoirs surnaturels (on s'attend à ce qu'il soit généreux, ses pouvoirs individuels étant donc réorientés vers la survie de la communauté) et en partie pour ritualiser (et donc pour minimiser) la possibilité qu'une hiérarchie politique se concrétise. Autrement dit, la force augmentée de l'individu se manifeste uniquement par sa générosité et non dans un rôle politique explicite. La générosité est signe concret de la force augmentée du chasseur qui, ironiquement, diminue le potentiel d'agir de l'homme fort; c'est donc un mécanisme qui reconnait tacitement la force surnaturelle du chasseur, sans pourtant lui permettre d'exercer explicitement son pouvoir sur le plan politique.

Le mystique est donc une instance de pouvoir qui touche certains individus (pas tous) ${ }^{17}$, augmentant leur efficacité et leur force. Ceci, évidemment, suscite des commentaires favorables (l'individu est plus généreux) et défavorables (l'individu est potentiellement surdoué et donc dangereux) de tout le monde, mais lance surtout une vague de commérages à propos des pouvoirs secrets du chasseur devenu "chanceux ", puisque les pouvoirs augmentés de l'individu sont cachés par une règle de silence qui interdit aux personnes de communiquer les détails de leurs rencontres avec la force primordiale. Parler serait trahir le secret de la conversation intime avec l'animal transcendantal; autrement dit, cela serait diluer le pouvoir en le partageant.

Loin d'augmenter le pouvoir politique de façon directe, cette règle du silence absolu, en ce qui concerne le contact qui est censé être à la base de leur force, crée une hiérarchie éphémère et ambiguë. Le silence formel et le commérage informel sont les seules façons de réconcilier la «surindividualité » de ces hommes marqués par le pouvoir mystique avec le besoin de solidariser la communauté. Loin d'attirer une attention positive, de définir une personnalité potentiellement charismatique qui mobiliserait la solidarité sociale à la manière de Weber, de tels individus sont évités. Ils n'occupent pas une position politique formelle qui pourrait fragmenter davantage une communauté fragile et toujours émergente, mais la crainte de leur pouvoir cumulée au respect et à la gratitude que l'on témoigne aux chasseurs chanceux agissent pour conférer une autorité morale à ces hommes qui, maintenant libérés des soucis relatifs à leur bien-être, par leur force acquise,

17. L'homme lance une invitation à l'Animal en adoptant l'apparence de gibier symbolique, mais l'Animal, comme un chasseur humain, ne prend pas toujours son gibier. 
peuvent se pencher sur des problèmes qui touchent la communauté entière. En fait, de tels hommes ont souvent une conscience sociale plus développée que leurs pairs. Ce sont eux que l'on écoute en ce qui concerne la question du déplacement des groupes de chasse, car, étant des chasseurs "chanceux ", leurs conseils sont respectés.

La générosité publique et le silence individuel (accompagnés par le commérage lui aussi «silencieux» à sa façon) finissent par créer une hiérarchie informelle dont les gradins supérieurs sont occupés par un ou deux individus doués d'une autorité morale incontestable attestée par des gestes extraordinaires, souvent de générosité, mais parfois de force physique prodigieuse. Ces individus président à la répartition des groupes de chasse autrement autonomes. Cette répartition du territoire rend possible la constitution d'une véritable communauté politique capable d'exprimer une volonté collective de posséder un territoire national. Bref, le silence entourant le contact avec le mystique permet aux Sekani de conserver intact l'individualité ontologiquement reconnue et valorisée, car la surpuissance individuelle à la base de l'agir est sémiotiquement déplacée du quotidien vers la dimension mystique qui définit la vraie intimité de chacun.

Le silence crée un espace ritualisé et sémantiquement appauvri dans le présent, où le pouvoir sémiotique des signes s'accroît par le commérage «silencieux" et rend en même temps plus «bruyant » l'indicible qui est à la base de l'organisation sociale ${ }^{18}$, c'est-à-dire les pouvoirs individuels qui sont à la base de la collaboration dans la chasse. L'harmonie sociale que les anthropologues voient souvent dans les sociétés de chasse n'est qu'une fiction utopique qui cache les tensions «silencieuses» et inévitables, puisqu'elle est issue de la nécessité de trouver une formule pour contraindre l'individu à se déplacer. Il n'est pas surprenant que toute décision politique engage les chasseurs dans une discussion censée mener à un consensus (une formule politique inévitable, étant donné la crainte populaire de l'individu contaminé par la force primordiale). Le consensus est un moyen efficace de faire taire la voix individuelle et de conserver intacte la structure sociale et ses tensions dynamiques: le silence individuel et le consensus public mènent non seulement au partage et donc à l'affaiblissement de la responsabilité individuelle, mais ils accroissent aussi le pouvoir de la voix collective en la

18. Ces termes sont inspirés, évidemment, par la discussion de Lévi-Strauss (1964, 327-328). 
plaçant dans l'espace rituel du non-dit mystique, puisque le consensus est tellement ritualisé par le silence qu'il devient dépourvu de signifiés concrets ${ }^{19}$.

\section{Conclusion}

En guise de conclusion, je voudrais faire la synthèse des trois étapes de la ritualisation du rapport individuel à la dimension mystique qui agit par les glissements métonymiques, aboutissant dans la création de l'image de la communauté politiquement solidaire en dépit de la dispersion de ses membres : 1) l'obligation qu'ont les groupes de chasse de se déplacer sur l'ensemble du territoire crée un champ rituel limité, dont les deux signes uniques qui le composent, les animaux et l'Homme, sont inversés, puisque l'espace sémiotique du champ d'action des animaux passe à l'Homme toujours en mouvement et donc métaphorisé en animal (la nature devient culture) ; 2) en revanche, le jeûne, l'isolement et surtout l'immobilité imposée sur les chasseurs en quête de contact avec les Animaux-protecteurs transforment symboliquement l'Homme en gibier et les Animaux-protecteurs en humains (la culture devient nature); 3) l'ensemble des rapports individualisés avec les Animaux devient un signifié dont le signifiant est caché

19. Le silence entourant le contact avec la dimension mystique transparaît dans le système traditionnel de nominalisation de l'espace topographique, qui attache les individus à des lieux particuliers, permettant aux personnes de "parler» des exploits des autres de façon transversale; par exemple, un lieu n'est pas connu par ses caractéristiques écologiques, mais par le nom d'un individu protagoniste d'un événement important ou extraordinaire. Évidemment, les toponymes anthropomorphisés ne sont pas inconnus en Europe (Rome; Barcelone), mais pour les Sekani, il semble qu'il s'agisse de leur seul système. Les défunts deviennent donc des métaphores du passé, mais l'événement extraordinaire par lequel ils sont connus devient métonymie du présent. Attribuer des noms d'individus aux lieux topographiques divorce l'espace de ses caractéristiques naturelles et transforme l'ensemble de l'espace humanisé en métonymie de l'individualité dont la surpuissance mystique s'est manifestée dans l'événement extraordinaire. En humanisant l'espace, la terre des individus devient donc métaphore de la communauté politique parce qu'il est métonymie de l'Animal transcendantal. Paradoxalement, l'humanisation de l'espace topographique, en faisant référence aux pouvoirs extraordinaires de certains individus, affaiblit l'idée de l'individualité et de la capacité humaine d'agir, renforçant l'idée selon laquelle la terre est un lieu éternel où vivent non seulement les animaux, mais aussi les Animaux-protecteurs de l'époque du Transformateur. Autrement dit, le passé vit toujours sous forme indirecte dans le présent, comme le mystique n'est aucunement une dimension séparée du quotidien. 
par le silence qui l'entoure; les pouvoirs transcendantaux des Animauxprotecteurs deviennent le signifié silencieux du discours politique, transformant ainsi, par la ritualisation de l'immobilité de la quête de vision, les conseils pratiques du chef à propos du déplacement des groupes de chasse en signifié de la communauté dont les membres sont toujours en mouvement. En fait, il n'est pas surprenant que des chasseurs formellement obligés de suivre le gibier utilisent ce trait fondamental pour définir les rapports avec la dimension mystique toujours dangereuse, qui aboutissent à la création de la communauté politique. Enfin, il est à souligner qu'au niveau le plus général, les Sekani procèdent, apparemment de façon paradoxale, à la création de la communauté en augmentant l'individualité de chacun. C'est par les conséquences de leur croyance en ces forces incontrôlables et dangereuses de la dimension mystique que la communauté naît, par le silence censé taire l'envie et ritualiser la contamination d'un individu devenu surpuissant. La crainte selon laquelle la dimension mystique peut anéantir l'individu mène à une vision de la communauté toujours émergente et toujours fragile, mais politiquement solidaire, sans nuire à l'individualité.

\section{Références}

BIERHORST, J. (1985), The Mythology of North America, New York, QuillWilliam Morrow.

Codrington, R. (1972 [1891]), The Melanesians, Oxford, Clarendon Press.

GoulET, J.-G. (1994), "Récits de rêves et de visions chez les Dénés Tha contemporains. Vision du monde et principes épistémologiques sousjacents ", Anthropologie et Sociétés, 18/2, p. 59-74.

Helm, J. (1994), Prophecy and Power among the Dogrib Indians, Lincoln, University of Nebraska Press.

HultKRANTZ, Åke (1967), Les religions des Indiens primitifs de l'Amérique, Stockholm, Almqvist and Wiksell (Stockholm Studies in Comparative Religion, 4).

Lanoue, G. (1992), Brothers: The Politics of Violence among the Sekani of Northern British Columbia, Oxford, Berg Publishers Ltd.

Lanoue, G. et N. Ferrara (2004), «Windigo "Psychosis”, Healing the Sick: Balancing the Self in Northern Canadian Hunting Societies", Anthropologica, 46/1, p. 69-83. 
Legros, D. (1999), Tommy McGinty's Story of Crow: A First Nations Elder Recounts the Creation of the World, Ottawa, Musée canadien des civilisations.

LÉvi-STrauss, C. (1964), Le cru et le cuit, Paris, Plon. (1973), «Le geste d'Asdival », Anthropologie structurale, II, Paris, Plon, p.175-233.

Ridington, R. (1988), "Knowledge, Power, and the Individual in Subarctic Hunting Societies ", American Anthropologist, 90/1, p. 98-110.

SHARP, H. S. (1987), "Giant Fish, Giant Otters and Dinosaurs, "Apparently Irrational Beliefs" in a Chipewyan Community ", American Ethnologist, 14/2, p. 226-235.

(1988), The Transformation of Big Foot: Maleness, Power and Belief among the Chipewyan, Washington, Smithsonian Institution Press.

Turner, D. H. (1978), «Ideology and Elementary Structures ", Anthropologica, 20/1-2, p. 223-247.

\section{Résumé}

Les Sekani de la Colombie septentrionale (Canada) étaient des chasseurs nomades qui vivaient, jusqu'aux années 1970, en petits groupes dispersés et autonomes sur le plan économique. Dans cet article, j'examine comment l'hyperindividualité qui accompagne ce style de vie est utilisée pour définir un champ de silence qui devient la base de la communauté politique. Pour ce faire, les Sekani déplacent l'agir individuel vers un domaine apparemment "surnaturel ", encourageant les chasseurs à prendre contact avec les esprits tutélaires. Le silence qui entoure de tels contacts déclenche des commérages «secrets » qui aboutissent à l'identification d'un homme fort capable ainsi d'assumer le statut de chef gestionnaire du territoire national.

\section{Abstract}

The Sekani of northern British Columbia (Canada) are nomadic hunters who, until the 1970s, lived in small, geographically dispersed and economically autonomous groups. In this paper, I analyse the role played by the hyper-individuality intrinsic to this lifestyle in creating a discursive zone of silence that becomes the foundation for defining the political community. In so doing, the Sekani project individual agency onto an apparently 
"supernatural" realm, encouraging hunters to enter into contact with tutelary spirits. The silence that surrounds such contacts unleashes "secret" rumours that ultimately result in the identification of a strong man thus deemed capable of donning the role of administrative leader ("chief") of the national territory. 